\title{
PTERIDOFLORA DO REFÚGIO ECOLÓGICO CHARLES DARWIN (IGARASSU, PERNAMBUCO, BRASIL) ${ }^{1}$
}

\author{
Augusto César Pessôa Santiago² \\ Iva Carneiro Leão Barros ${ }^{2}$
}

Recebido: 25/08/2002. Aceito: 03/06/2003

\begin{abstract}
RESUMO - (Pteridoflora do Refúgio Ecológico Charles Darwin (Igarassu, Pernambuco, Brasil)). Foi realizado o levantamento florístico das pteridófitas ocorrentes no Refúgio Ecológico Charles Darwin, município de Igarassu, Pernambuco, Brasil. A área é um remanescente de Floresta Atlântica que está em processo de preservação há aproximadamente 40 anos. Visando contribuir para o conhecimento das pteridófitas e seus aspectos ecológicos em Pernambuco, foram realizadas 13 excursões, no período de um ano e meio, para observação e coleta das plantas, acompanhando as estações chuvosa e seca. Foram registradas 21 espécies, distribuídas em 16 gêneros e 12 famílias: Thelypteridaceae (5 spp.), Polypodiaceae (3 spp.), Schizaeaceae (3 spp.), Blechnaceae (2 spp.), Aspleniaceae (1sp.), Cyatheaceae (1 sp.), Davalliaceae (1 sp.), Dennstaedtiaceae (1 sp.), Dryopteridaceae (1 sp.), Pteridaceae (1sp.), Selaginellaceae (1 sp.) e Vittariaceae (1 sp.). Foi registrada uma nova referência para o Nordeste do Brasil: Schizaea subtrijuga Mart. Predominam as espécies herbáceas, terrícolas, hemicriptófitas, ciófilas, encontradas principalmente no interior da mata. Apesar da maioria das espécies registradas serem amplamente distribuídas no Estado, a presença de espécies não muito frequentes em áreas de Floresta Atlântica em Pernambuco, como Cyclodium meniscioides (Willd.) C. Presl, Salpichlaena volubilis (Kaulf.) J. Sm., Polypodium aureum L. var. aureum e Selaginella dendricola Jenman e o registro de uma nova referência para o Nordeste, evidenciam a importância desta pteridoflora.
\end{abstract}

Palavras-chave - Pteridófitas, florística, Mata Atlântica, Nordeste do Brasil, Pernambuco

\begin{abstract}
Pteridoflora of the "Refúgio Ecológico Charles Darwin" (Igarassu, Pernambuco, Brazil)). This paper presents a floristic survey of the pteridophytes of the "Refúgio Ecológico Charles Darwin", located at the municipality of Igarassu, Pernambuco State, Brazil. This area is a remnant of the Atlantic forest, which has been preserved during the last 40 years. In order to contribute to the knowledge of the ferns and its ecological traits in Pernambuco, 13 field expeditions were made during a period of one year and a half. Thus observations and collection of plants were carried out, during the rainy and the dry seasons. Twenty one species were registered, distributed in 16 genera and 12 families: Thelypteridaceae (5 spp.), Polypodiaceae (3 spp.), Schizaeaceae (3 spp.), Blechnaceae (2 spp.), Aspleniaceae (1 sp.), Cyatheaceae (1 sp.), Davalliaceae (1 sp.), Dennstaedtiaceae (1 sp.), Dryopteridaceae (1 sp.), Pteridaceae (1 sp.), Selaginellaceae (1 sp.) and Vittariaceae (1 sp.). It was registered a new reference for the northeastern Brazil: Schizaea subtrijuga Mart. The local pteridophyte species are preferentially
\end{abstract}

1 Monografia de conclusão do Curso de Graduação do primeiro Autor

2 Departamento de Botânica, Programa de Pós-Graduação em Biologia Vegetal, CCB, Universidade Federal de Pernambuco, Av. Prof. Moraes Rego, s/n, Cidade Universitária, CEP 50560-901, Pernambuco, Brasil (augustosantiago@hotmail.com; ivaleao@truenet.com.br) 
herbaceous, terrestrial, hemicryptophyte, inhabiting the interior of the forest, as ciophilous. Despite most of the species is widely distributed in the state, the occurrence of species not frequently registered in remnants of Atlantic forest in Pernambuco, as Cyclodium meniscioides (Willd.) C. Presl, Salpichlaena volubilis (Kaulf.) J. Sm., Polypodium aureum L. var. aureum and Selaginella dendricola Jenman, and the new reference for the northeastern Brazil enhance the importance of this pteridoflora.

Key words - Pteridophytes, floristic, Atlantic forest, Northeastern Brazil, Pernambuco State

\section{Introdução}

No Estado de Pernambuco as pteridófitas ocorrem preferencialmente nas áreas de Floresta Atlântica, assim como nas regiões Sudeste e Sul do Brasil (Ambrósio \& Barros 1997; Labiak \& Prado 1998). Mesmo assim, algumas espécies são encontradas nas regiões semi-áridas de caatinga no Nordeste do Brasil (Barros et al. 1989). Segundo Tryon \& Tryon (1982), cerca de $65 \%$ das espécies de pteridófitas ocorrem em ambientes tropicais úmidos.

A literatura cita número de espécies variando de 9.000 a 12.000 , das quais cerca de 3.250 ocorrem nas Américas (Tryon \& Tryon 1982; Windisch 1990). Destas, aproximadamente $30 \%$ podem ser encontradas em território brasileiro, que abriga inclusive um dos centros de endemismo e especiação de pteridófitas no continente (Tryon 1972).

Os estudos realizados com as pteridófitas para a região Nordeste do Brasil, tiveram início com Spring (1840), Sturm (1859), Baker (1870) e Kuhn (1884), quando realizaram suas coletas no país e cujas espécies estão descritas na "Flora Brasiliensis" de Martius \& Eichler (1840-1884). Atualmente, dos nove Estados que compõem a região Nordeste do Brasil a pteridoflora é melhor conhecida em Pernambuco, Alagoas, Ceará e Paraíba. Trabalhos direcionados com estudos de pteridófitas para os Estados do Maranhão, Piauí, Rio Grande do Norte, Sergipe e Bahia são pouco conhecidos (Barros 1996).

Barros (1997) apresentou listagem das espécies referidas para o Estado. Posteriormente foram desenvolvidos os trabalhos de Silva (2000) na Mata do Estado, município de São Vicente Férrer e no local do presente trabalho. Nestes trabalhos são referidas cerca de 300 espécies de pteridófitas para Pernambuco, das quais cerca de $80 \%$ ocorrem em áreas de Mata Atlântica.

O conhecimento dos grupos vegetais e animais é de extrema importância para os planos de conservação e necessário para aplicação dos projetos de manejo e preservação das espécies. Assim, esse trabalho teve como objetivo contribuir com o conhecimento da pteridoflora na Floresta Atlântica de Pernambuco, pois apesar de numerosos estudos já terem sido realizados nos remanescentes locais, muitas são as áreas que ainda não foram analisadas do ponto de vista florístico, sendo necessário conhecê-las para obter-se mais dados sobre a composição e distribuição deste grupo.

\section{Material e métodos}

Caracterização da área de estudo - O Estado de Pernambuco situa-se na região Nordeste do Brasil e sua vegetação encontrase dividida em quatro zonas fitogeográficas: Litoral, Mata, Caatinga e Savanas. Devido a sua maior extensão no sentido Leste-Oeste, o Estado apresenta ampla diversidade de formações vegetacionais o que reflete considerável diversidade de sua pteridoflora (Andrade-Lima 1961; Barros 1997). A Zona da Mata ou Floresta Atlântica representa um ponto de ligação das florestas orientais brasileiras, que vêm do sul, com as florestas equatoriais brasileiras, que vêm da Amazônia, e ocupa aproximadamente 15,7\% da área total do Estado de Pernambuco (Andrade-Lima 1960; 1961).

A Zona da Mata é dividida em três subzonas: Mata Úmida, Mata Seca e Mata Serrana 
(Andrade-Lima 1960). Dentro da subzona de Mata Úmida está o Refúgio Ecológico Charles Darwin, que se localiza no município de Igarassu, litoral Norte do Estado de Pernambuco e apresenta as seguintes coordenadas geográficas: latitude $07^{\circ} 48^{\prime} 37^{\prime \prime}$ e $07^{\circ} 49^{\prime} 2^{\prime}$ 'S e longitude $34^{\circ} 27^{\prime} 25^{\prime}$ ' e $34^{\circ} 56^{\prime} 52^{\prime \prime}$ W. A área é cortada pelo rio Jacoca, também chamado de Tabatinga, que é perene e faz parte da principal bacia hidrográfica do município, a do rio Botafogo. $\mathrm{O}$ local apresenta uma trilha principal que serve para entrada de carros, e trilhas acessórias destinadas às caminhadas dos visitantes no interior da mata.

Deve-se considerar que as áreas florestais do município de Igarassu vêm sendo bastante degradadas, ao longo dos anos, com o desmatamento para fins agropecuários, o que acontece com as áreas em torno do Refúgio. Contudo, os 60ha da área de estudo estão em processo de recomposição, devido a medidas conservacionistas (Neves 1998).

A vegetação é típica da Zona da Mata, uma Formação Florestal Perenifólia Latifoliada Higrófila Costeira. O clima é do tipo Am's segundo Köppen, com transição para o tipo As. A temperatura média anual é de $27^{\circ} \mathrm{C}$, com umidade relativa do ar em torno de $80 \%$ (Andrade-Lima 1960; 1961). A mata é formada por um estrato arbóreo variando entre 8 e $15 \mathrm{~m}$ de altura, e um estrato herbáceo-arbustivo.

Coleta, identificação e herborização - Foram realizadas 13 excursões ao Refúgio Ecológico Charles Darwin no período de novembro/1998 a abril/2000, com a finalidade de observação das espécies e de realização das coletas, acompanhando os períodos climáticos diferenciados no Estado de Pernambuco.

As identificações foram realizadas com auxílio de bibliografia especializada, principalmente Alston et al. (1981), Proctor (1985), Tryon \& Tryon (1982), Moran \& Riba (1995), Fernandes (1997), Salino (2000), Sylvestre (2001). O sistema de classificação adotado está de acordo com a ordenação filogenética de Tryon \& Tryon (1982), exceto pela adição dos gêneros Macrothelypteris (H. Itô) Ching (Thelypteridaceae) e Cyclodium (Willd.) C. Presl (Dryopteridaceae), baseados em Smith (1992) e Smith (1986), respectivamente. Os nomes dos autores foram atualizados segundo Pichi-Sermolli (1996).

O material-testemunho foi depositado no Herbário UFP (Prof. Geraldo Mariz, Universidade Federal de Pernambuco), com duplicatas para o Herbário PEUFR (Prof. Vasconcelos Sobrinho, Universidade Federal Rural de Pernambuco) e doações ao Herbário SI (Instituto Botánico Darwinion, San Isidoro, Buenos Aires, Argentina).

Aspectos ecológicos - Foram feitas observações, em campo, sobre alguns aspectos ecológicos das pteridófitas presentes na área de estudo. Foram anotados os tipos de hábito, habitat, a forma de vida, os tipos de ambiente $\mathrm{e}$ os ambientes preferenciais, baseados na metodologia de Barros (1997) e Ambrósio \& Barros (1997), com algumas modificações.

\section{Resultados e discussão}

Em relação à riqueza florística das pteridófitas ocorrentes no Refúgio Ecológico Charles Darwin, esta compreende flora com 21 espécies, 16 gêneros e 12 famílias (Tab. 1). Para as Matas Úmidas de Pernambuco, são referidas 250 espécies de pteridófitas, enquanto nas Matas Serranas são registradas 127 e em Matas Secas, 97 espécies (Barros 1997; Silva 2000). Entre as espécies encontradas no Refúgio, exceto Schizaea subtrijuga, que constitui nova referência, todas já haviam sido citadas para o Estado. Esta espécie já era registrada para a região Norte (Takeuchi 1960) e para as regiões Sul e Sudeste (Brade 1972). A espécie apresentou várias populações no local, com grande número de indivíduos, principalmente em bordos de trilhas e interior da mata, em locais úmidos e parcialmente sombreados.

A família mais representativa no local do 
Tabela 1. Riqueza de espécies de pteridófitas do Refúgio Ecológico Charles Darwin, município de Igarassu, Estado de Pernambuco, Brasil.

Família/Espécies

ASPLENIACEAE

Asplenium serratum $\mathrm{L}$.

BLECHNACEAE

Blechnum serrulatum Rich.

Salpichlaena volubilis J. Sm.

CYATHEACEAE

Cyathea microdonta (Desv.) Domin

DAVALLIACEAE

Nephrolepis biserrata (Sw.) Schott

DENNSTAEDTIACEAE

Pteridium aquilinum (L.) Kuhn

DRYOPTERIDACEAE

Cyclodium meniscioides (Willd.) C. Presl

POLYPODIACEAE

Microgramma vacciniifolia (Langsd. \& Fisch.) Copel.

Polypodium aureum L. var. aureum

P. decumanum Willd.

PTERIDACEAE

Pityrogramma calomelanos (L.) Link

SCHIZAEACEAE

Lygodium venustum $\mathrm{Sw}$.

L. volubile $\mathrm{Sw}$.

Schizaea subtrijuga Mart.*

SELAGINELLACEAE

Selaginella dendricola Jenman

THELYPTERIDACEAE

Macrothelypteris torresiana (Gaudich.) Ching

Thelypteris dentata (Forsk.) E.P. St. John

T. hispidula (Decne.) C.F. Reed

T. interrupta (Willd.) K. Iwats.

T. serrata (Cav.) Alston

VITTARIACEAE

Vittaria lineata (L.) Sm.
Coletor (n.)

Santiago 46

Santiago 07,10

Santiago 09

Santiago 27, 30

Santiago 01,35,37

Santiago 13,38, 24

Santiago 06,16,18

Santiago 26,40

Santiago 22

Santiago 29, 32

Santiago 12

Santiago 02

Santiago 19

Santiago 48, 49

Santiago 30, 34, 28

Santiago 14, 24, 33

Santiago 21, 25, 41

Santiago 03, 15, 17

Santiago 04

Santiago 05

Siqueira s/n

\footnotetext{
* Nova referência para o Nordeste do Brasil
}

presente estudo foi Thelypteridaceae, com cinco espécies, seguida de Polypodiaceae e Schizaeaceae, com três espécies cada. Segundo Smith (1992), Thelypteridaceae é família subcosmopolita, com muitas espécies de ocorrência em regiões tropicais e subtropicais do mundo. As Thelypteridaceae foram representadas apenas por espécies terrícolas. A família Polypodiaceae, segundo Moran (1995), compreende principalmente espécies epífitas, ocasionalmente terrícolas ou rupícolas, é cosmopolita, grande e diversificada. Das espécies de Polypodiaceae registradas no Refúgio três se apresentaram como epífitas e apenas uma como terrícola. As Schizaeaceae representam uma família primitiva, com quatro gêneros que apresentam alta diversidade em relação ao hábito e forma (Tryon \& Stolze 1989).

Com respeito aos aspectos ecológicos estudados nas espécies que compõem a flora 
do Refúgio Ecológico Charles Darwin, as pteridófitas são predominantemente herbáceas, terrícolas, ciófilas, higrófilas e mesófilas; apresentam a forma de vida hemicriptófita em sua grande maioria; crescem e se desenvolvem preferencialmente no interior da mata (Tab. 2). Nota-se que há plena concordância com os estudos realizados por Barros (1997) para a flora pteridofítica da Floresta Atlântica em Pernambuco.

Nephrolepis biserrata é a espécie que apresenta maior distribuição no local, estando presente em quase todas as categorias de ambientes preferenciais registradas neste trabalho, apresentando as formas de vida hemicriptófita ou epífita. Em alguns locais do Refúgio esta espécie foi coletada próxima a Lygodium volubile e L. venustum em bordos de trilhas; associada a Thelypteris hispidula no interior da mata e em outro ponto, à T. dentata e Macrothelypteris torresiana.

Pteridium aquilinum foi considerada heliófila, sendo encontrada no bordo da mata em local exposto ao sol, em ambiente bastante seco, no limite da área do Refúgio e vizinha a uma plantação.

No bordo da mata, em local parcialmente sombreado e paludoso durante o período de chuvas, crescem próximas as espécies Macrothelypteris torresiana, Thelypteris interrupta, T. serrata e Blechnum serrulatum.

Em ambiente de interior de mata (a cerca de $3 \mathrm{~m}$ distante da trilha principal), em local sombreado e paludoso, foram observadas pequenas populações de Nephrolepis biserrata, Macrothelypteris torresiana e Thelypteris dentata crescendo junto a várias angiospermas herbáceas. Cyathea microdonta foi encontrada próxima ao curso do regato, às vezes associada com Thelypteris hispidula e Nephrolepis biserrata, em bordas de trilhas e no interior da mata em terreno paludoso, próxima às espécies Cyclodium meniscioides e Salpichlaena volubilis.

Algumas espécies terrícolas apresentaram poucos indivíduos isolados em locais sombreados, como Polypodium aureum var. aureum, Pityrogramma calomelanos e Selaginella dendricola. Em relação às espécies epífitas, Polypodium decumanum foi coletada associada a Microgramma vacciniifolia, em clareira. A primeira foi coletada a cerca de $1,5 \mathrm{~m}$ do solo até os ramos mais altos e a segunda, em uma ramificação a cerca de $1 \mathrm{~m}$ até os ramos mais altos. Asplenium serratum foi coletada a altura aproximada de $1,5 \mathrm{~m}$ e Vittaria lineata, a cerca de $2 \mathrm{~m}$.

A maioria das espécies encontradas no local são amplamente distribuídas no Estado e comuns em áreas de Mata Úmida, de acordo com estudos realizados no Estado, como os de Fonseca (1992), Farias et al. (1992), Barros et al. (1995-1996), Ambrósio \& Barros (1997) e Silva (2000). Estas espécies são também normalmente observadas em áreas com visível degradação. Duas destas podem ser consideradas introduzidas em território brasileiro (Macrothelypteris torresiana e Thelypteris dentata), mas são subespontâneas na América tropical (Smith 1992) e também são bastante comuns no Estado de São Paulo (Salino 2000). Outras espécies são pouco encontradas nas áreas de Mata Úmida no Estado e só foram registradas em áreas mais preservadas, como a Mata do Estado em São Vicente Férrer (Silva 2000), como Salpichaena volubilis e Cyclodium menisciodes, enquanto Polypodium aureum var. aureum e Selaginella dendricola só são referidas para áreas de Brejo de Altitude (Mata Serrana).

O número de espécies encontradas é comparável ao de outras áreas no Estado, como as Reservas Ecológicas de Caetés (Barros et al. 1995; 1996; Farias et al. 1992) e Jangadinha (Ambrósio \& Barros 1997), com 16 e 25 espécies, respectivamente. Porém este número pode ser considerado pequeno se comparado com as áreas detentoras de maior riqueza no Estado, como por exemplo a Reserva Ecológica de Gurjaú, com 56 espécies (Fonseca 1992) e a 
Tabela 2. Aspectos ecológicos observados nas espécies de pteridófitas do Refúgio Ecológico Charles Darwin, município de Igarassu, Estado de Pernambuco, Brasil. Hábito: $\mathrm{H}=$ herbáceo; $\mathrm{A}=$ arborescente; $\mathrm{T}=$ trepadeira. Habitats: $\mathrm{C}=$ corticícola; $\mathrm{T}=$ terrícola. Ambientes preferenciais: $\mathrm{CL}=$ clareira, $\mathrm{BM}=$ bordo de mata, $\mathrm{BR}=$ barranco, $\mathrm{IM}=$ interior de mata, $\mathrm{LP}=$ locais paludosos, $\mathrm{MT}=$ margem de trilha, $\mathrm{MR}=$ margem de regato. Formas de vida: $\mathrm{E}=$ epífita; $\mathrm{C}=$ caméfita; $\mathrm{H}=$ hemicriptófita; $\mathrm{G}$ = geófita. Tipos de ambiente: $\mathrm{M}=$ mesófila; $\mathrm{C}=$ ciófila; $\mathrm{H}$ = higrófila.

Espécies

Hábito Hábitat Ambientes preferenciais Formas de vida Tipos de ambiente

\begin{tabular}{|c|c|c|c|c|c|}
\hline Asplenium serratum & $\mathrm{H}$ & $\mathrm{C}$ & IM & $\mathrm{E}$ & M \\
\hline Blechnum serrulatum & $\mathrm{H}$ & $\mathrm{T}$ & BM, MR e LP & $\mathrm{C}$ & $\mathrm{M}, \mathrm{C}$ e $\mathrm{H}$ \\
\hline Cyathea microdonta & A & $\mathrm{T}$ & IM, MT, MR e LP & $\mathrm{C}$ & $\mathrm{Ce} \mathrm{H}$ \\
\hline Cyclodium meniscioides & $\mathrm{H}$ & $\mathrm{T}$ & IM, MR e LP & $\mathrm{H}$ & $\mathrm{Ce} \mathrm{H}$ \\
\hline Lygodium venustum & $\mathrm{T}$ & $\mathrm{T}$ & IM, BR e MT & $\mathrm{H}$ & $\mathrm{M}$ e $\mathrm{C}$ \\
\hline L. volubile & $\mathrm{T}$ & $\mathrm{T}$ & IM, BR e MT & $\mathrm{H}$ & $\mathrm{M}$ e $\mathrm{C}$ \\
\hline Macrothelypteris torresiana & $\mathrm{H}$ & $\mathrm{T}$ & IM, BM e LP & $\mathrm{H}$ & $\mathrm{Ce} \mathrm{H}$ \\
\hline Microgramma vacciniifolia & $\mathrm{H}$ & $\mathrm{C}$ & IM, MBM, MT e CL & $\mathrm{E}$ & $\mathrm{Me} \mathrm{C}$ \\
\hline Nephrolepis biserrata & $\mathrm{H}$ & $\mathrm{Ce} \mathrm{T}$ & IM, BR, BM, MT e CL & $\mathrm{HeE}$ & $\mathrm{M}$ e $\mathrm{C}$ \\
\hline Pityrogramma calomelanos & $\mathrm{H}$ & $\mathrm{T}$ & IM, MT & $\mathrm{H}$ & $\mathrm{C}$ e H \\
\hline Polypodium aureum var. aureum & $\mathrm{H}$ & $\mathrm{T}$ & IM & $\mathrm{H}$ & $\mathrm{Me} \mathrm{C}$ \\
\hline P. decumanum & $\mathrm{H}$ & $\mathrm{C}$ & IM, BR, MT e CL & $\mathrm{E}$ & $\mathrm{M}$ e $\mathrm{C}$ \\
\hline Pteridium aquilinum & $\mathrm{H}$ & $\mathrm{T}$ & $\mathrm{BM}$ & $\mathrm{G}$ & $\mathrm{H}$ \\
\hline Salpichlaena volubilis & $\mathrm{T}$ & $\mathrm{T}$ & IM, MR e LP & $\mathrm{H}$ & $\mathrm{C}$ e H \\
\hline Schizaea subtrijuga & $\mathrm{H}$ & $\mathrm{T}$ & IM e MT & $\mathrm{H}$ & M \\
\hline Selaginella dendricola & $\mathrm{H}$ & $\mathrm{T}$ & IM, BR e MT & $\mathrm{H}$ & $\mathrm{Ce} \mathrm{H}$ \\
\hline Thelypteris dentata & $\mathrm{H}$ & $\mathrm{T}$ & IM e LP & $\mathrm{H}$ & $\mathrm{C}$ e $\mathrm{H}$ \\
\hline T. hispidula & $\mathrm{H}$ & $\mathrm{T}$ & IM e MR & $\mathrm{H}$ & $\mathrm{C}$ e $\mathrm{H}$ \\
\hline T. interrupta & $\mathrm{H}$ & $\mathrm{T}$ & BM e LP & $\mathrm{C}$ & $\mathrm{Me} \mathrm{H}$ \\
\hline T. serrata & $\mathrm{H}$ & $\mathrm{T}$ & BM e LP & $\mathrm{H}$ & $\mathrm{Me} \mathrm{H}$ \\
\hline Vittaria lineata & $\mathrm{H}$ & $\mathrm{C}$ & IM & $\mathrm{E}$ & M \\
\hline
\end{tabular}

Mata do Estado, com 94 espécies (Silva 2000). Apesar da devastação que aconteceu no entorno da área estudada, o Refúgio Ecológico Charles Darwin ainda apresenta as condições ambientais preservadas, evidenciadas pela presença de espécies pouco encontradas nas áreas de Matas Úmidas do Estado de Pernambuco e uma nova referência para a região Nordeste. Estes dados refletem a importância da pteridoflora local, que provavelmente deve ter sido mais rica anteriormente, quando não havia ação antrópica.

\section{Agradecimentos}

Os autores agradecem ao Biólogo Roberto Siqueira, proprietário do Refúgio Ecológico Charles Darwin, pela permissão, apoio e incentivo ao estudo; à Dra. Monica Ponce, pela confirmação da espécie Thelypteris hispidula; ao Dr. Alexandre Salino, pela determinação de Thelypteris dentata; à Dra. Eliana Simabukuro e à Bióloga Flávia Silva, pelo apoio nas excursões; à Dra. Ariadna Lopes, pela revisão do Abstract.

\section{Referências bibliográficas}

Alston, A. H. G.; Jermy, A. C. \& Rankin, J. M. 1981. The genus Selaginella in Tropical South America. Bulletin of the British Museum (Natatural History) Botany 4(9): 233-330.

Ambrósio, S. T. \& Barros, I. C. L. 1997. Pteridófitas de uma área remanescente de Floresta Atlântica do Estado de Pernambuco, Brasil. Acta Botanica Brasilica 11(2): 105-113.

Andrade-Lima, D. 1960. Estudos fitogeográficos de Pernambuco. Arquivos do Instituto de Pesquisas Agronômicas 5: 305-341. 
Andrade-Lima, D. 1961. Tipos de Florestas de Pernambuco. Anais da Associação de Geógrafos Brasileiros 12: 69-85.

Baker, J. G. 1870. Cyatheaceae et Polypodiaceae. In: C. E. P. Martius \& A. G. Eichler (eds.). Flora Brasiliensis. Lipsiae apud Frid. Fleischer in Comm. Monachii, 1(2): 306-624.

Barros, I. C. L. 1996. Pteridófitas. Pp. 111-121. In: E. V. S. B. Sampaio; S. J. Mayo \& M. R. V. Barbosa (eds.). Pesquisa Botânica Nordestina: Progresso e Perspectivas. Sociedade Botânica do Brasil, Seção Regional de Pernambuco.

Barros, I. C. L. 1997. Pteridófitas ocorrentes em Pernambuco: ensaio biogeográfico e análise numérica. Tese de Doutorado. Universidade Federal Rural de Pernambuco, Recife.

Barros, I. C. L.; Fonseca, E. R.; Valdevino, J. A. \& Paula, E. L. 1995-1996. Contribuição ao estudo taxonômico das pteridófitas ocorrentes na Reserva Ecológica de Caetés (Paulista, PE) Thelypteridaceae. Dryopteridaceae. Davalliaceae. Polypodiaceae. Lycopodiaceae. Boletim da Sociedade Broteriana Sér. 2, 67: 271-286.

Barros, I. C. L.; Silva, A. J. R. \& Silva, L. L. S. 1989. Levantamento florístico das pteridófitas ocorrentes na Zona das Caatingas do Estado de Pernambuco, Brasil. Biologica Brasilica 1(2): 143-159.

Brade, A. C. 1972. Contribuição ao conhecimento das espécies brasileiras do gênero Schizaea, com especial referência às espécies do Brasil Austral. Boletim do Herbarium Bradeanum 1(26): 285-295.

Farias, M. C. A.; Belo, M. A. M. \& Barros, I. C. L. 1992. Pteridófitas da Reserva de Caétes (Paulista, PE). Boletim da Sociedade Broteriana. Sér. 2, 65 : 149-162.

Fernandes, I. 1997. Taxonomia e fitogeografia de Cyatheaceae e Dicksoniaceae nas regiões Sul e Sudeste do Brasil. Tese de Doutorado. Universidade de São Paulo, São Paulo.

Fonseca, E. R. 1992. Pteridófitas da Reserva do Gurjaú, Cabo, Pernambuco. Dissertação de Mestrado. Universidade Federal de Pernambuco, Recife.

Kuhn, M. 1884. Isoetaceae et Salviniaceae. In: C. F. P. Martius \& A. G. Eichler (eds.). Flora Brasiliensis. Lipsiae apud Frid. Fleischer in Comm. Monachii, 1(2): 246-662.

Labiak, P. H. \& Prado, J. 1998. Pteridófitas epífitas da Reserva Volta Velha, Itapoá, Santa Catarina, Brasil. Boletim do Instituto de Botânica 11: 1-79.
Moran, R. C. 1995. Polypodiaceae. In: Pp. 359-363. G. Davidse; M. Sousa \& S. Knapp (eds.). Flora Mesoamericana. Vol.1 - Psilotaceae a Salviniaceae. México: Universidad Nacional Autónoma de México.

Neves, R. M. L. 1998. Estudo de ectoparasitos de aves silvestres (Passeiriformes: Emberezidae) de Mata Atlântica, Igarassu, Pernambuco. Dissertação de Mestrado. Universidade Federal de Pernambuco, Recife.

Pichi-Sermolli, R. E. G. 1996. Authors of scientific names in Pteridophyta. Royal Botanical Garden, Kew.

Proctor, G. R. 1985. Ferns of Jamaica: A guide to Pteridophytes. British Museum Natural History, London.

Salino, A. 2000. Estudos taxonômicos da família Thelypteridaceae (Polypodiopsida) no Estado de São Paulo, Brasil. Tese de Doutorado. Universidade Estadual de Campinas, Campinas.

Silva, M. R. 2000. Pteridófitas da Mata do Estado, Serra do Mascarenhas, município de São Vicente Férrer, Estado de Pernambuco, Brasil. Dissertação de Mestrado. Universidade Federal de Pernambuco, Recife.

Smith, A. R. 1986. Revision of the Neotropical fern genus Cyclodium. American Fern Journal 76(2): 56-98.

Spring, A. F. 1840. Lycopodiaceae. In: C. F. P. Martius \& A. G. Eichler (eds.). Flora Brasiliensis. Lipsiae apud Frid. Fleischer in Comm. Monachii. 1(2): 106-136.

Sturm, J. G. 1859. Ophioglossaceae et Hymenophyllaceae. In: C. F. P. Martius \& A. G. Eichler (eds.). Flora Brasiliensis. Lipsiae apud Frid. Fleischer in Comm. Monachii. 1(2): 142-304.

Sylvestre, L. S. 2001. Revisão taxonômica das espécies de Aspleniaceae A.B. Frank ocorrentes no Brasil. Tese de Doutorado. Universidade de São Paulo, São Paulo.

Takeuchi, M. 1960. O gênero Schizaea na Amazônia. Boletim do Museu Paraense Emílio Goeldi 5: $1-25$.

Smith, A. R. 1992. Thelypteridaceae. In: R. M. Tryon \& R. G. Stolze. Pteridophyta of Peru. Part III. 16. Thelypteridaceae. Fieldiana Botany 29: 1-80.

Tryon, R. M. \& Stolze, R. G. 1989. Pteridophyta of Peru. Part I. 1. Ophioglossaceae - 12. Cyatheaceae. Fieldiana Botany 27: 1-145.

Tryon, R. M. 1972. Endemic areas and geographical speciation in tropical american ferns. Biotropica 4: 121-131. 
Tryon, R. M. \& Tryon, A. F. 1982. Ferns and allied plants with special reference to tropical America. Springer Verlag, New York.
Windisch, P. G. 1990. Pteridófitas da Região NorteOcidental do Estado de São Paulo - Guia para excursões. UNESP, São José do Rio Preto.

Versão eletrônica do artigo em www.scielo.br/abb 